physiologic salt solution, and heating from forty to sixty minutes at $60 \mathrm{C}$. (140 F.).

The case which we wish to report was treated by vaccines and the Roentgen ray. There was an intercurrent acute suppurative appendicitis.

History.-A retired farmer, aged 63, married, who had had rheumatism when a child, and occasional attacks of myalgia since, but no other illness, in the latter part of February, 1914, had what he described as "a breaking out on the face." A few days later he noticed swellings beneath the angles of the jaws and ears; these gradually increased in size, and in two or three weeks, swelling was noticeabie in the axilla and groins. The tumor formations were never painful, tender or red. He rapidly became weak and emaciated, and complained of extreme nervousness.

Examination.-Tumor formations as shown in Figure 1 were present. The masses apparently consisted of enlarged discrete, nonadherent, painless lymph nodes. The mucous membranes were pale. Physical examination otherwise was negative.

The urine was pale amber, acid, specific gravity 1.008 , albumin and sugar negative. Microscopically there were a few hyaline casts and amorphous urates. The red corpuscles in the blood numbered $3,200,000$; hemoglobin, from 55 to 60 ; white blood cells, 7,200; polymorphonuclears, 59; mononuclears, 41.

Treatment.-In June, 1914, one of the glands was taken from the neck and sent to Dr. F. W. Gaarde of Chicago for diagnosis and preparation of autogenous vaccine.

July 1, 1914, v a c c in e therapy was begun, consisting of subcutaneous injections twice a week for seven weeks. There were no severe reactions. The initial dose was 25,000,000 ; this was gradually increased to $2,000,000$,000 , and in addition to this the patient was given Roentgen-ray t $r$ e a tment every five days for eight weeks. He received six injections before there was any amelioration in the symptoms or the size of the glands. After this they gradually reduced to

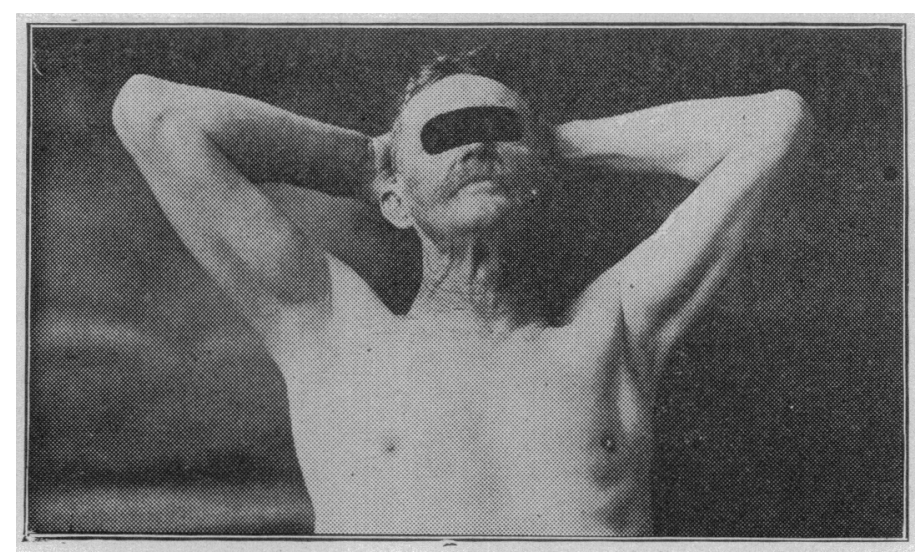

Fig. 2.-Patient, July 1, 1915, showing results of treatment.

\section{POLYPUS OF FALLOPIAN TUBE}

\author{
Lawrence H. Hoffman, M.D., San Francisco \\ Fellow of the American College of Surgeons
}

Polypus of the fallopian tube, although mentioned in textbooks of gynecology, is rarely encountered in pelvic surgery. The condition may exist without causing any symptoms and pass unrecognized until it reaches a size to be discovered by palpation during some routine pelvic examination or until the occurrence of rupture of the containing fallopian tube demands operative procedure.

The case I report is interesting on account of its rarity and its simulation of ectopic gestation. I have searched the literature and can find no cases of that nature on record.

History.-The patient, aged 30, married several years, presented herself to Dr. Oscar Mansfeldt with the following history. She had never become pregnant although she had used no preventive measures. Menstruation had always been regular, moderate in quantity, about four days' duration. The April and May menses were normal. The June 1 period was on time but patient menstruated several days longer than usual, with a day's cessation, and a few days of a dark bloody discharge with clots, accompanied by colicky pains in the lower abdomen and slight abdominal distention.

Examination, June 18.-The cervix was conical, of normal consistency. The uterus was normal in size, in axis of pelvis not fixed. A boggy semifluctuating mass was palpable in Douglas' culdesac. The right adnexa was palpable and no abnormality was felt. A mass was felt in the left parametrium about $5 \mathrm{~cm}$. in diameter, immobile, separable from the uterus and tender to the touch. The findings were corroborated by an examination under $n$ a r cos is June 18 , and I was called in consultation to verify the findings. A diagnosis of ectopic gestation was made and operation was decided on and performed June 19.

Operation. - When the perito neal cavity was opened, by median abdomi-

about two thirds of their original size. August 27, he was taken ill with acute suppurative appendicitis. He was operated on, September 8 , and an abscess was drained. The tubes were removed on the eighth day, and drainage ceased about the eighteenth. During this time there was an extremely rapid decrease in the size of the glands. With cessation of pus there was a corresponding slackening in the retrogression of the glands which did not cease entirely, but has continued until the present time.

Constitutionally, except for a dragging sensation at the site of the scar, the patient feels perfectly well, and is doing his own chore work on the farm.

The question arises as to what influence the pus infection had on the glands. There is no doubt that the toxins from infectious processes at times exercise a profound effect on newly formed tissue, as illustrated by the use of Coley's toxins in sarcoma. The fact, however, that the glands were decreasing under the vaccine treatment, and continued to do so after the infection had cleared up, and that the results are identical with those of Billings and Rosenow, leads us to believe that while the appendicitis may have been a favoring influence, the main results were due to the vaccines. nal incision, some fluid, blood and clots escaped. The left adnexa was represented by a mass $5 \mathrm{~cm}$. in diameter, embedded in blood clots, and ruptured on its superior margin near its distal end. This adnexa was removed typically and the clots evacuated from the peritoneal cavity. The alodomen was closed in four layers. Recovery was uneventful.

The pathologic report, made by Dr. Ophüls, was of rupture of the fallopian tube, which contained a polypus, 2 $\mathrm{cm}$. in diameter, and coagula.

When discussing the case, the existence of ectopic gestation was weighed but the fact that there had been no amenorrhea and that uterine enlargement was absent caused a doubt in our minds. The other symptoms and physical signs were so suggestive of extra-uterine pregnancy that we felt an exploratory operation was demanded, knowing the dangers of such a condition. Until the pathologist's report was received the findings were taken as ectopic pregnancy, and great was our surprise when the true nature of the lesion was disclosed.

530 Butler Building.

Preventive Medicine.-Within the past thirty years in this country the mortality from tuberculosis has been reduced more than half, and with scarlet fever and diphtheria the results have been more striking. Within the past ten years the average life has been increased four years.-Vaughan. 